\title{
EEG Signal Extraction Method Based on HHT and CSP
}

\author{
Lei Gong, Chengzhi Liu \\ Tianjin University of Science and Technology, China; \\ E-mail:*1442064639@qq.com \\ www.tust.edu.cn
}

\begin{abstract}
In this paper, the Hilbert Huang transform is used to extract the marginal spectrum of the four-task motor imagery of EEG signals. Firstly, a spatial filter constructed by common spatial pattern is used to extract the feature of signal marginal spectrum. The MI EEG Data2a provided by The University of GRAZ is verified by the experiment, and the results show that compared with the traditional single CSP feature extraction method, the effect of MSCSP feature extraction was significantly improved.
\end{abstract}

Keywords: motor imagery (MI), Hilbert Huang transform (HHT), common spatial pattern (CSP), marginal spectrum (MS)

\section{Introduction}

Electroencephalogram (EEG) is to record the spontaneous and rhythmic activity of brain cells through electrodes. EEG based brain computer interface (BCI) system does not depend on muscle nerve activity, but on EEG activity recorded at scalp ${ }^{1}$. As a new interdisciplinary subject, BCI technology has attracted the attention and research of many international and domestic researchers, and has shown broad application prospects in the fields of rehabilitation medicine engineering, auxiliary control and military ${ }^{2}$. With the rapid development of science and technology, although the quality of BCI technology has been improved rapidly, there is still a certain distance between BCI technology and practical application, among which the search for excellent feature extraction method is one of the keys.

The brain computer interface based on $\mathrm{MI}$ is a commonly used experimental paradigm of spontaneous EEG signals. The basic principle of MI is that when people imagine a certain limb of the body moving, according to the previous motor memory, the motor cortex of the brain will have corresponding potential changes ${ }^{3}$. Imagining hands, feet and tongue is a typical four-task in MI brain computer interface. EEG signal processing and analysis is the core part of the whole BCI system. Feature extraction is mainly through the analysis of EEG signal in time domain, frequency domain and spatial domain to find the features which are difficult to be found in the original EEG signal and can best reflect the thinking activity ${ }^{4}$. The existing feature extraction methods mainly include power spectral density (PSD), independent component analysis (ICA), discrete wavelet transform (DWT), Hilbert Huang (HHT) and common space pattern (CSP) $)^{5,6,7,8}$.

In this paper, the marginal spectrum (MS) of four-task motor imagery of EEG is extracted by Hilbert Huang transform (HHT). For the first time, the common spatial pattern (CSP) is used to construct a spatial filter to extract the marginal spectral features (MSCSP) of the signals. Combining with the classification method of linear discriminant analysis (LDA), the classification accuracy of EEG signals is improved, which creates conditions for the practical application of BCI system.

\section{Experimental Data}

(C) The 2021 International Conference on Artificial Life and Robotics (ICAROB2021), January 21 to 24,2021 
The data set selected in this paper is the BCI competition IV dataset2a motor imagery data set in the fourth brain computer interface competition in 2008 , provided by Graz University of science and technology, Austria ${ }^{9}$. The data set was collected from 9 subjects. Each subject performed four-task motor imagery tasks: left hand, right hand, double feet, and tongue. Each subject was given two experiments. The EEG data collected for the first time is used as training set, and the task tag is known. The EEG data collected in the second time is used as the test set, and the task tag is unknown. In each data set, the subjects conducted 6 rounds of experiments, and 48 groups of data were collected in each round. Therefore, a total of 288 groups of experimental data were collected for each subject ${ }^{10}$.

The whole process of the experiment is shown in Fig.1.The first stage is the preparation stage, i.e., when $\mathrm{t}=$ 0 s, a cross symbol appears on the screen and a sound prompts the subject to prepare to start the experiment; the second stage is the motor imagery task prompt stage, that is, when $t=2 s$, a task prompt arrow appears on the screen. At this time, the subjects were reminded to start to prepare for the motor imagery task; the third stage was the motor imagery stage, that is, when $\mathrm{t}=3 \mathrm{~s}$, the motor imagery task was started, lasting for $6 \mathrm{~s}$ until the cross symbol on the screen disappeared. The fourth stage is the rest stage. After a short interval, the next round of experiment is carried out.

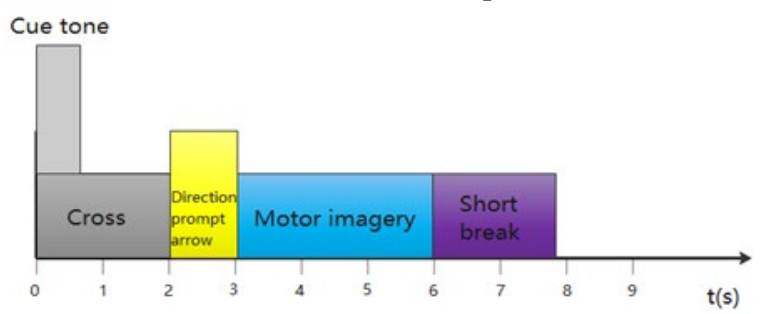

Fig.1. Experimental flowchart

\section{Hilbert Huang Transform}

HHT includes two parts: Empirical Mode Decomposition (EMD) and Hilbert transform ${ }^{11,12}$. It retains the advantages of time-frequency local transform in discrete wavelet transform and overcomes the problem of no adaptive basis function. This method of adaptive EEG decomposition does not necessarily require automatic signal processing.

After HHT transformation, the three-dimensional spectrum of time amplitude frequency is obtained. As shown in Fig.2, the three-dimensional spectrum of an EEG signal after HHT transformation can obtain the timefrequency information of EEG signal more visually.

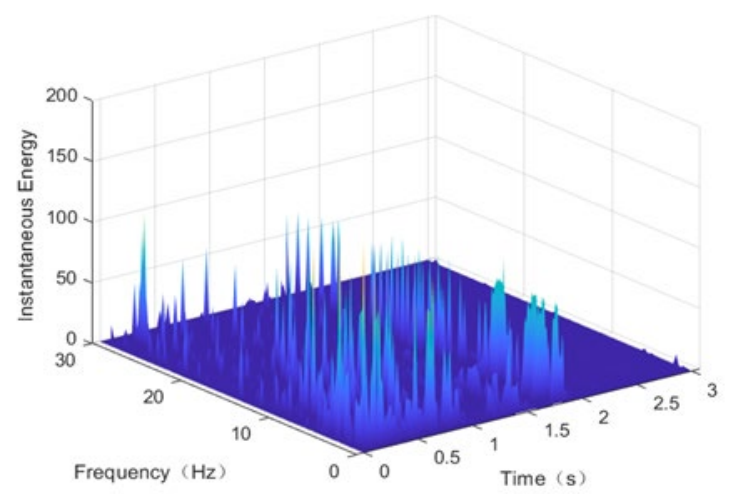

Fig.2. Three-dimensional spectrum of EEG signal after HHT transformation

\section{Improved CSP Algorithm Based on Marginal Spectrum}

The traditional CSP algorithm is operated directly in the time domain signal. It is difficult to train a good adaptability projection matrix on account of the complexity of EEG signal time-domain waveform change. When the brain carries out motor imagery, obvious ERD and ERS phenomena will appear in the $\mathrm{C} 3, \mathrm{C} 4$ and $\mathrm{CZ}$ regions of the cerebral cortex ${ }^{13}$. This phenomenon will cause obvious changes in the amplitude of the rhythm (8$13 \mathrm{hz}$ ) and $\beta$ rhythm (14-25hz) of the signals in this region. This frequency phenomenon has more obvious differences than the time-domain signals. Therefore, this paper proposes a method combining CSP algorithm with signal marginal spectrum (MS) algorithm (MSCSP). The algorithm combines the good binary classification effect of CSP algorithm and the good time-frequency characteristics of the marginal spectrum, so the MSCSP feature matrix has better adaptability.

\subsection{Marginal spectrum feature extraction}

In order to obtain the marginal spectrum, EMD decomposition is used to divide the complex signal into simple IMF single component signal, so that the concept of instantaneous frequency can be applied. Then the instantaneous frequency is analyzed by Hilbert transform, and the Hilbert spectrum is integrated with time to obtain the Hilbert marginal spectrum ${ }^{14,15}$. Therefore, MS is defined as follows:

(C) The 2021 International Conference on Artificial Life and Robotics (ICAROB2021), January 21 to 24,2021 


$$
h(w)=\int_{0}^{T} H(w, t) d t
$$

Where $\mathrm{T}$ is the length of the signal. four-task of motor imagery data, including left hand, right hand, double feet, and tongue, are selected from the standard data set, and the marginal spectra of $\mathrm{C} 3, \mathrm{C} 4$ and $\mathrm{CZ}$ channel data of these four tasks are extracted by HHT transformation, as shown in Fig. 3 .

It is obvious from the Fig. 3 that when the subjects are imagining the movement of their left and right hands, feet and tongue, obvious ERD and ERS phenomena appear in C3, C4 and CZ channels, and the amplitude of the edge spectrum changes with the change of frequency in the whole frequency band. Although it is similar to the spectrum, its meaning is different from that of the frequency spectrum, which represents the value of each frequency amplitude in the whole data set. It can accurately reflect the actual frequency component of the signal. The amplitude of a point in the spectrum proves that there is a trigonometric function of the frequency in the whole signal. The larger the amplitude, the higher the matching degree between the trigonometric function of the frequency and the original signal. By comparing the difference between the marginal spectrum and Fourier transform, it can be found that the marginal spectrum can not only process the stationary signal, but also the nonstationary signal. We can sensitively find the energy transformation of a certain frequency in the signal. So the marginal spectrum can reflect the actual frequency component of the signal more accurately.
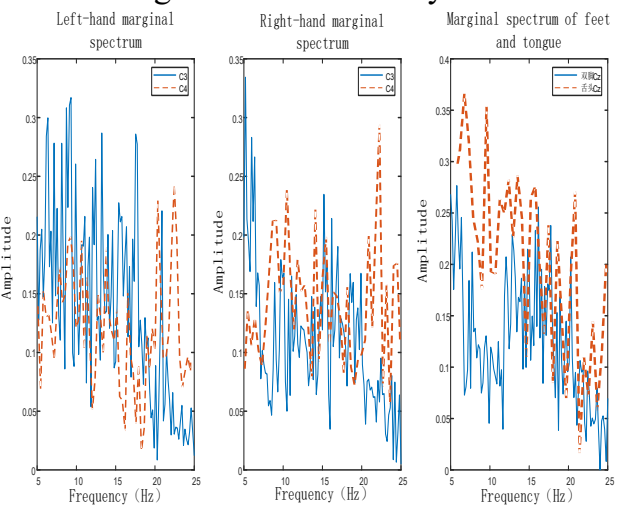

Fig.3. Marginal Spectra of Four Kinds of Motion Imagination

\subsection{Improved algorithm of CSP}

In this paper, the "one-to-one" CSP feature extraction method is used to process four kinds of task motor imagery EEG signals. In this paper, four-task of motor imagery signals are divided into two groups (left hand 1vs right hand 2 , left hand $1 \mathrm{vs}$ feet 3 , left hand $1 \mathrm{vs}$ tongue 4 , right hand $2 \mathrm{vs}$ feet 3 , right hand $2 \mathrm{vs}$ tongue 4 , feet $3 \mathrm{vs}$ tongue 4). Six sets of CSP features are obtained by constructing six spatial filters through CSP, which are used as input of classifiers and the final output category.

After preprocessing the motor imagery data of the training set $4 \mathrm{~S}$, the marginal spectrum of each channel is obtained by HHT transformation. The CSP projection matrix and training set features are obtained by CSP operation of the marginal spectrum of each channel. For the test set data, after the same preprocessing, the marginal spectrum of each channel is obtained by HHT transformation, and the test set characteristics can be obtained by multiplying it with the CSP projection matrix.

The MSCSP characteristics of EEG signals before and after marginal spectrum processing are shown in Fig.4, in which Fig.4(a) is the six groups of CSP feature maps without obtaining the marginal spectrum by HHT transformation, and Fig.4 (b) is the six groups of MSCSP feature maps with marginal spectrum obtained by HHT transformation. From the feature map, it can be found that the CSP features of left hand and right hand, left hand and tongue, right hand and tongue, and tongue and feet before transformation have small distance and feature overlap seriously, so they can not be well distinguished. After the marginal spectrum is obtained by HHT transform, the interval between the two kinds of MSCSP features increases and the feature overlap decreases, which can distinguish the two kinds of signals well. Therefore, the marginal spectrum EEG signal processing method based on HHT transform can improve the discrimination of MSCSP features and ensure the accuracy of classification.
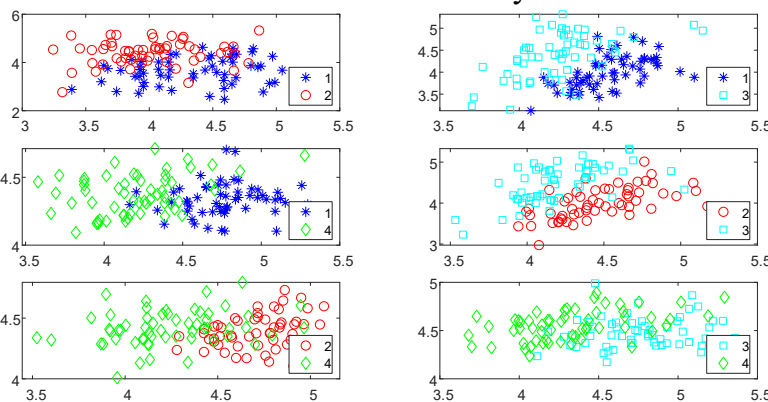

(a)

C. The 2021 International Conference on Artificial Life and Robotics (ICAROB2021), January 21 to 24, 2021 

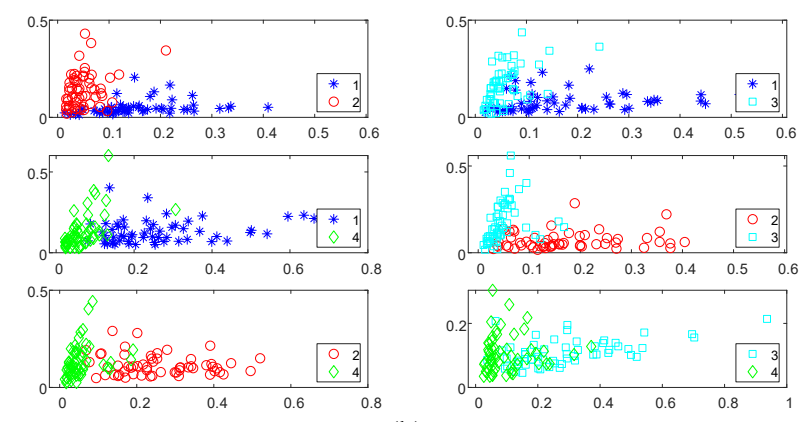

(b)

Fig.4. (a) CSP feature map before marginal spectrum processing, (b) MSCSP feature map before marginal spectrum processing

\section{Analysis of Experimental Results}

After preprocessing the BCI competition IV dataset $2 \mathrm{a}$ data set, the EEG signals were divided into four groups according to the motor imagery tasks of left hand, right hand, double feet and tongue. Each group contained 72 single experiments. In each experiment, the EEG signal components during motor imagery, i.e., the data from $3 \mathrm{~s}$ to $6 \mathrm{~s}$, were intercepted, and the power spectral density (PSD), discrete wavelet coefficients (DWT), common space pattern features (CSP) and the improved algorithm MSCSP were extracted respectively. SVM classifier was used for feature classification. The final classification results are shown in Table 1.

Table 1. Comparison of classification accuracy of features extracted by each algorithm

\begin{tabular}{lllll}
\hline \multirow{2}{*}{ Subjects } & \multicolumn{4}{c}{ Feature extraction method (accuracy\%) } \\
\cline { 2 - 5 } A01T & 44.83 & DWT & CSP & MSCSP \\
A02T & 43.38 & 48.72 & 71.43 & 67.86 \\
A03T & 41.38 & 68.97 & 53.57 & 57.14 \\
A04T & 20.69 & 41.38 & 50.89 & 83.93 \\
A05T & 43.10 & 20.69 & 33.57 & 58.93 \\
A06T & 25.86 & 31.03 & 42.14 & 41.07 \\
A07T & 46.55 & 51.72 & 68.21 & 55.36 \\
A08T & 48.83 & 55.17 & 80.18 & 67.86 \\
A09T & 44.83 & 48.28 & 63.75 & 66.14 \\
average & $39.93 \pm 9.77$ & $46.36 \pm 14.01$ & $60.05 \pm 15.98$ & $64.48 \pm 12.61$ \\
\hline
\end{tabular}

From the final classification results in Table 1, under the same preprocessing conditions, it can be found that the method of extracting PSD of signal correlation frequency band has the lowest classification effect. Secondly, DWT is used to extract low-frequency coefficients of signals, which has no good classification effect. In time domain, the effect of CSP feature extraction is much better than the first two methods. However, compared with the improved
MSCSP algorithm, the final classification result is still relatively low. Therefore, compared with the other three algorithms, the improved MSCSP algorithm can extract EEG features and has great advantages.

\section{Conclusion}

In the BCI system based on motor imagery, effective feature extraction and classification is the key to the system. This paper improves the CSP algorithm, uses HHT to extract the marginal spectrum of EEG signals, and selects the marginal spectrum as the input to extract the features of CSP. Based on the data provided by GRAZ University, the paper analyzes the feature extraction effect of the improved MSCSP algorithm, and finds it is better than the traditional CSP algorithm. The feature extraction method in this paper is in line, it is with the current development direction of $\mathrm{BCI}$, and provides an experimental basis for application of BCI system.

\section{Acknowledgements}

\section{References}

1. Blankertz B , Tomioka R, Lemm S , et al. Optimizing spatial filters for robust EEG single-trial analysis. IEEE Signal Processing Magazine, 2007, 25(1): 41-56.

2. Emanuel N, Felix B , Harald A , et al. Regularized Linear Discriminant Analysis of EEG Features in Dementia Patients. Frontiers in Aging Neuroence, 2016, 8: 273-.

3. Haas, L F. Hans Berger (1873-1941), Richard Caton (1842-1926), and electroencephalography. J Neurol Neurosurg Psychiatry, 2003, 74(1): 9.

4. Vidal Toward direct brain-computer communication. Annual Re- view of Biophysics and Bioengineering, 1973, 2(1): 157.

5. Tiwari N, Edla DR, Dodia S, et al. Brain computer interface: A comprehensive survey. Biologically Inspired Cognitive Architectures, 2018, 26: 118-129.

6. Dong E Z, Li C H, Li L T, et al. Classification of multi-class motor imagery with a novel hierarchical SVM algorithm for brain-computer interfaces. Medical \& Biological Engineering \& Computing, 2017, 55(10): 1809-1818

7. Jeunet $\mathrm{C}$, Lotte $\mathrm{F}$, Batail $\mathrm{J} \mathrm{M}$, et al. Using recent $\mathrm{BCI}$ literature to deepen our understanding of clinical neurofeedback: A short review. Neuroscience, 2018, 378: 225-233.

8. Naeem M, Brunner C, Leeb R, et al. Seperability of four-class motor imagery data using independent

(C) The 2021 International Conference on Artificial Life and Robotics (ICAROB2021), January 21 to 24, 2021 
components analysis. Journal of neural engineering, 2006, 3(3): 208

9. Naeem M, Brunner C, Leeb R, et al. Seperability of four-class motor imagery data using independent components analysis. Journal of neural engineering, 2006, 3(3): 208.

10. Pfurtscheller G, Neuper C, Guger C, et al. Current trends in Graz Brain-Computer Interface (BCI) research. IEEE Trans Rehabil Eng, 2000, 8(2): 216219.

11. Dong E , Zhou K , Tong J , et al. A novel hybrid kernel function relevance vector machine for multi-task motor imagery EEG classification. Biomedical Signal Processing and Control, 2020, 60: 101991.

12. Bauschke H H , Combettes P L . Convex Analysis and Monotone Operator Theory in Hilbert Spaces. 2011, 10. 1007/978-1-4419-9467-7.

13. Liu C , Zhao H B , Li C S , et al. Classification of ECoG motor imagery tasks based on CSP and SVM 2010 3rd International Conference on Biomedical Engineering and Informatics. IEEE, 2010.

14. Yeo M V M , LiX , Shen K , et al. Can SVM be used for automatic EEG detection of drowsiness during car driving. Safety Science, 2009, 47(1): 115-124.

15. Vladimir Cherkassky and Yunqian Ma. Practical selection of SVM parameters and noise estimation for SVM regression. Neural Networks, 2004. 